\title{
Wireless adiabatic power transfer
}

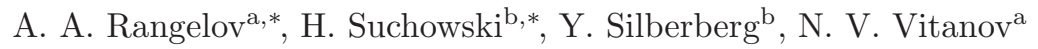 \\ ${ }^{a}$ Department of Physics, Sofia University, James Bourchier 5 blvd., 1164 Sofia, Bulgaria \\ ${ }^{b}$ Department of Physics of Complex System, Weizmann Institute of Science, Rehovot \\ 76100, Israel
}

\begin{abstract}
We propose a technique for efficient mid-range wireless power transfer between two coils, by adapting the process of adiabatic passage for a coherently driven two-state quantum system to the realm of wireless energy transfer. The proposed technique is shown to be robust to noise, resonant constraints, and other interferences that exist in the neighborhood of the coils.

Keywords: Wireless energy transfer, Adiabatic passage, Robust and efficient power transfer, Coupled mode theory.

PACS: 05.45.Xt, 32.80.Xx, 84.32.Hh, 85.80.Jm
\end{abstract}

\section{Introduction}

The search for wireless power transfer techniques is as old as the invention of electricity. From Tesla, trough the vast technological development during the 20th century till recent days, many proposals have been made and implemented in this research field. Established techniques for wireless energy transfer are known both in the near- and far-field coupling regimes. Examples for the former can be found in resonant inductive electric transformers [1], optical waveguides 2] and cavity couplers [3]. In the far field, one can find the mechanism of transferring electromagnetic power by beaming a light source to a receiver which is converted to usable electrical energy [4]. Although these techniques enable sufficiently efficient energy transfer, they suffer either from the short-range interaction in the near-field, or from the requirement of line of sight in the far-field approaches. Recently, it was shown that weakly radiative wireless energy transfer between two identical classical resonant coils is possible with sufficiently high efficiency [5, 6, 6]. This breakthrough was made possible by the application of the coupled mode theory into the realm of power transfer. In this experiment, Kurs et. al. [5] showed that energy can be transferred wirelessly at distances of about 2 meters (mid-range) with efficiency of about $40 \%$.

\footnotetext{
*These authors contributed equally to this work

Email address: rangelov@phys.uni-sofia.bg (A. A. Rangelov)
} 
Currently, most efficient wireless energy transfer devices rely upon the constraint of exact resonance between the frequencies of the emitter (the source) and the receiver (the device, or the drain) coils [5, 6, 7]. When the frequency of the source is shifted from the frequency of the device, due to lack of similarity between the coils or by random noise (introduced, for example, by external objects placed close to either coils), a significant reduction of the transfer efficiency would occur. In such case, one may implement a feedback circuit, as suggested in Ref. [5], in order to correct the reduction of the transfer efficiency.

In this paper, we suggest a different approach to resolve the issues of the resonant energy transfer process. Here, we present a novel technique for robust and efficient mid-range wireless power transfer between two coils, by adapting the process of adiabatic passage (AP) for a coherently driven two-state quantum system [8, 9], as will be explained in the following sections. The adiabatic technique promises to be both efficient and robust for variations of the parameters driving the process, such as the resonant frequencies of the coils and the coupling coefficient between them.

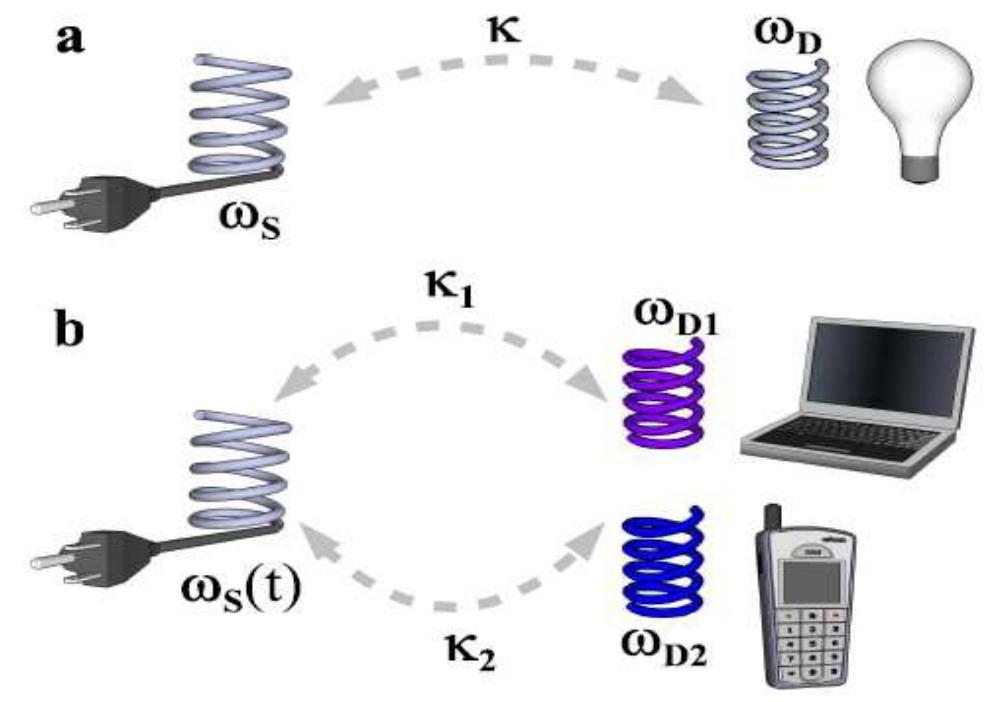

Figure 1: (Color online) Methods for wireless energy transfer. (a) For constant coil frequencies, efficient transfer from the source to a device occurs only when $\omega_{S}=\omega_{D}$. (b) The proposed adiabatic technique, with time-varying coil frequency $\omega_{S}(t)$, can transfer energy to multiple devices even when most of the time $\omega_{S}(t) \neq \omega_{D_{1}}, \omega_{D_{2}}$.

We follow the description of the coupled mode theory in the context of wireless energy transfer as described in detail by Kurs et. al. [5]. The interaction 
between two coils, in the strong-coupling regime, is described by the coupledmode theory, through the following set of two differential equations [3, 5]:

$$
i \frac{\mathrm{d}}{\mathrm{d} t}\left[\begin{array}{c}
a_{S}(t) \\
a_{D}(t)
\end{array}\right]=\left[\begin{array}{cc}
\omega_{S}(t)-i \Gamma_{S} & \kappa(t) \\
\kappa(t) & \omega_{D}(t)-i \Gamma_{D}-i \Gamma_{W}
\end{array}\right]\left[\begin{array}{c}
a_{S}(t) \\
a_{D}(t)
\end{array}\right] .
$$

Here, $a_{S}(t)$ and $a_{D}(t)$ are defined so that the energies contained in the source and the drain are, respectively, $\left|a_{S}(t)\right|^{2}$ and $\left|a_{D}(t)\right|^{2} . \Gamma_{S}$ and $\Gamma_{D}$ are the intrinsic loss rates (due to absorption and radiation) of the source and the drain coils, respectively, and the extraction of work from the device is described by the term $\Gamma_{W}$. The intrinsic frequencies of the source and drain coils are $\omega_{S}(t)$ and $\omega_{D}(t)$; these are given explicitly as

$$
\omega_{m}(t)=1 / \sqrt{L_{m}(t) C_{m}(t)} \quad(m=S, D),
$$

where $L_{S, D}(t)$ and $C_{S, D}(t)$ are the inductance and the capacitance, respectively, of the source and the drain coils. The coupling coefficient between the two coils reads

$$
\kappa(t)=M(t) \sqrt{\frac{\omega_{S}(t) \omega_{D}(t)}{L_{S}(t) L_{D}(t)}},
$$

where $M(t)$ is the mutual inductance of the two coils. The source coil is a part of the driving circuit and is periodically recharged, while the energy is transferred wirelessly to the device coil. The dynamics of such process in the case of static (time independent) resonance frequencies, as describe in Ref. [5] is illustrated in Fig. 1 (top).

The evolution of Eq. (11) is connected to the dynamics of the Schrödinger equation for a two-state atom written in the rotating-wave approximation [8, 9]. The variables $a_{S}(t)$ and $a_{D}(t)$ can be identified as the probability amplitudes for the ground state (corresponding to the source) and the excited state (corresponding to the drain), respectively. The coupling between the coils are analogous to the coupling coefficient of the two-state atom (also known as the Rabi frequency), which is proportional to the atomic transition dipole moment $\mathbf{d}_{12}$ and the laser electric field amplitude $\mathcal{E}(t): \Omega(t)=-\mathbf{d}_{12} \cdot \mathcal{E}(t) / \hbar[8,9]$. The difference between the resonant frequencies of the two coils corresponds to the detuning $\Delta(t)$ in the two-state atom: $\Delta(t)=\omega_{D}(t)-\omega_{S}(t)$.

The power transfer method was demonstrated for the resonant case of $\omega_{S}=$ $\omega_{D}=$ const, which is the case of $\Delta=0$ in atomic physics. However, the power transmitted between the coils drops sharply as the system is detuned from resonance, i.e. for the case of $\omega_{S} \neq \omega_{D}$. Also, any time dependent dynamics or change of coupling strengths between the coils can results in lower energy transfer between the coils.

\section{Adiabatic wireless energy transfer}

In the following, we develop a systematic framework of the adiabatic criteria in the context of wireless energy transfer. The technique of adiabatic passage 
was successfully implemented in other research fields, such as nuclear magnetic resonance (NMR) [10, 11], interaction of coherent light with two level atoms [8, 9], or in sum frequency conversion techniques in nonlinear optics [12, 13]. This dynamical solution requires a time-dependent intrinsic frequency change of the source coil $\omega_{S}(t)$. The variation of the frequency should be adiabatic (very slow) compared to the internal dynamics of the system that is determined by the coupling coefficient.

We will first assume that the loss rates $\Gamma_{S}, \Gamma_{D}$ and $\Gamma_{W}$ are zero and write Eq. (11) in the so-called adiabatic basis [8, 9] (for the two-state atom this is the basis of the instantaneous eigenstates of the Hamiltonian):

$$
i \frac{\mathrm{d}}{\mathrm{d} t}\left[\begin{array}{l}
b_{-}(t) \\
b_{+}(t)
\end{array}\right]=\left[\begin{array}{cc}
-\varepsilon(t) & i \dot{\vartheta}(t) \\
i \dot{\vartheta}(t) & \varepsilon(t)
\end{array}\right]\left[\begin{array}{l}
b_{-}(t) \\
b_{+}(t)
\end{array}\right],
$$

where the dot denotes a time derivative and

$$
\begin{aligned}
\varepsilon(t) & =\frac{1}{2} \sqrt{4 \kappa(t)^{2}+\Delta(t)^{2}}, \\
\tan 2 \vartheta(t) & =\frac{2 \kappa(t)}{\Delta(t)} .
\end{aligned}
$$

The connection between the original amplitudes $a_{S}(t)$ and $a_{D}(t)$ and the adiabatic ones $b_{-}(t)$ and $b_{+}(t)$ is given by

$$
\begin{aligned}
& b_{-}(t)=a_{S}(t) \cos \vartheta(t)-a_{D}(t) \sin \vartheta(t), \\
& b_{+}(t)=a_{S}(t) \sin \vartheta(t)+a_{D}(t) \cos \vartheta(t) .
\end{aligned}
$$

When the evolution of the system is adiabatic, $\left|b_{-}(t)\right|^{2}$ and $\left|b_{+}(t)\right|^{2}$ remain constant 14. Mathematically, adiabatic evolution means that the non-diagonal terms in Eq. (4) are small compared to the diagonal terms and can be neglected. This restriction amounts to the following adiabatic condition on the process parameters 8, 9]:

$$
|\dot{\kappa}(t) \Delta(t)-\kappa(t) \dot{\Delta}(t)| \ll\left[4 \kappa(t)^{2}+\Delta(t)^{2}\right]^{3 / 2} .
$$

Hence adiabatic evolution requires a smooth time dependence of the coupling $\kappa(t)$ and the detuning $\Delta(t)$, long interaction time, and large coupling and/or large detuning. In the adiabatic regime $\left|b_{ \pm}(t)\right|^{2}=$ const, but the energy contained in the source and the drain coil $\left|a_{S, D}(t)\right|^{2}$ will vary if the mixing angle $\vartheta(t)$ varies; thus adiabatic evolution can produce energy transfer between the two coils.

If the detuning $\Delta(t)$ sweeps slowly from some large negative value to some large positive value (or vice versa), then the mixing angle $\vartheta(t)$ changes from $\pi / 2$ to 0 (or vice versa). With the energy initially in the first coil, the system will stay adiabatically in $b_{-}(t)$ and thus the energy will end up in the second coil. Therefore the detuning sweep (i.e. the frequency chirp) will produce complete energy transfer. Furthermore, AP is not restricted to the shape of the coupling $\kappa(t)$ and the detuning $\Delta(t)$ as far as the condition (7) is fulfilled and the mixing angle $\vartheta(t)$ changes from $\pi / 2$ to 0 (or vice versa). 
The variation of the detuning $\Delta(t)$ can be achieved by changing the capacitance (or the inductance) of one, or the two coils. The time variation of the coupling $\kappa(t)$ can be achieved, for example, with the rotation of one coil (or two coils), thereby changing the geometry and thus the mutual inductance $M(t)$ of the two coils.
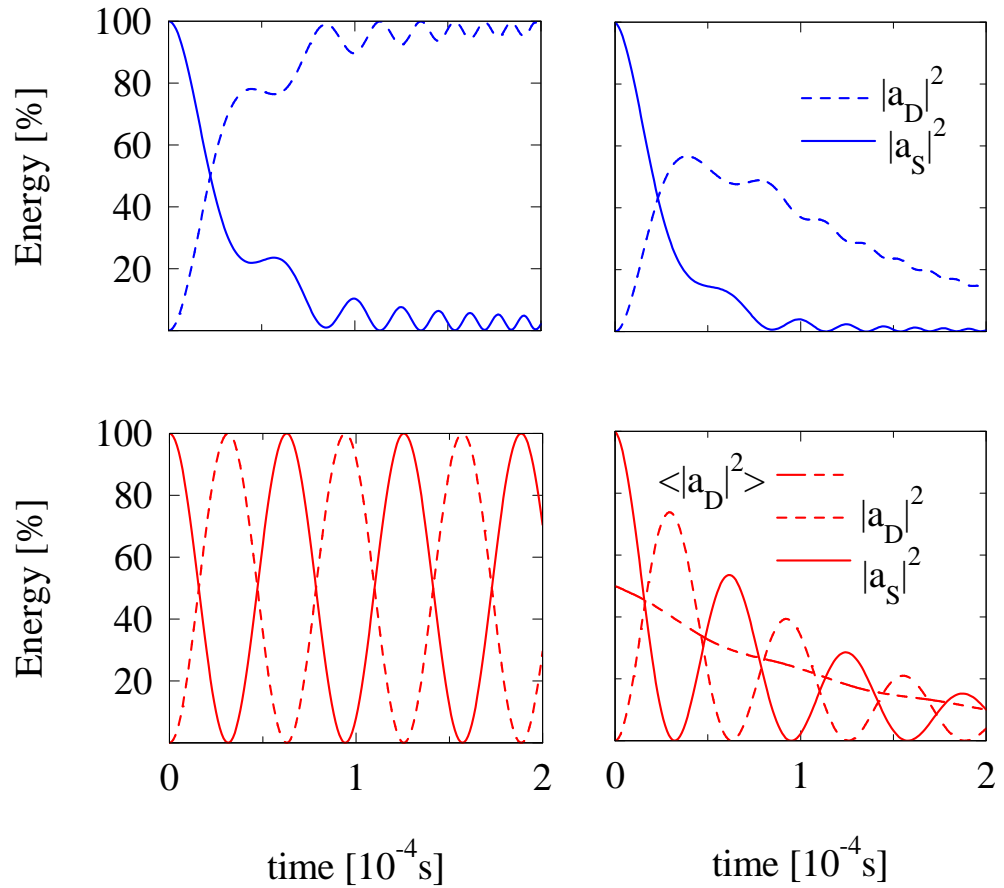

Figure 2: (Color online) Comparison between the energy transfer as a function of time for the AP case (top frames) and the static case (bottom frames), with no losses (left frames) and with loss $\Gamma_{S, D}=2 \times 10^{3} \mathrm{~s}^{-1}$ (right frames). In all the graphs, solid line refers to the source coil, and dashed line refers to the device coil. For the static case, the functions $\kappa(t)$ and $\Delta(t)$ are given by Eqs. (9) with the following parameters: $\kappa_{0}=4 \times 10^{4} \mathrm{~s}^{-1}, \delta=0 \mathrm{~s}^{-1}$, whereas for the AP case, they follow Eqs. (10) with the following parameters: $\kappa_{0}=4 \times 10^{4}$ $\mathrm{s}^{-1}, \delta=2 \times 10^{5} \mathrm{~s}^{-1}, \beta=3 \times 10^{9} \mathrm{~s}^{-2}$.

When the loss rates are nonzero, the dynamics become more complicated and more realistic. Nevertheless, the essence of AP remains largely intact, if one follow another important constraint,

$$
\Gamma_{S, D}<\kappa_{0}<|\Delta(t)| .
$$

which states that the coupling coefficient also should be larger than the loss rates, and that the initial and final detunings are larger than the coupling coefficient [8, 9]. The physical reasoning behind it is that the dynamics should be faster then the damping rates that exist in the system (mainly on the device) and not only adiabatic. In Fig. 2 we compare the resonant (static) and adiabatic 
mechanisms, without losses (left frames) and with losses (right frames). For the numerics, we used the following coupling and detuning for the resonant case:

$$
\begin{aligned}
\kappa(t) & =\kappa_{0}, \\
\Delta(t) & =\delta,
\end{aligned}
$$

and for the adiabatic mechanism:

$$
\begin{aligned}
\kappa(t) & =\kappa_{0}, \\
\Delta(t) & =\delta+\beta\left(t-t_{0}\right),
\end{aligned}
$$

where for our simulations we set $t_{0}=10^{-4} \mathrm{~s}$. As can be seen in Fig. 2 the energy in the static case oscillates back and forth between the two coils. In AP, once the energy is transferred to the drain coil it stays there. This feature of AP is used to minimize the energy losses from the source coil. We see that when following the adiabatic constraints, the AP process outperforms the static resonant method.

To describe the efficiency of the proposed technique we use the efficiency coefficient $\eta$, which is the ratio between the work extracted from the drain for the time interval $T$ divided by the total energy (absorbed and radiated) for the same time interval,

$$
\eta=\frac{\Gamma_{W} \int_{0}^{T}\left|a_{D}(t)\right|^{2} d t}{\Gamma_{S} \int_{0}^{T}\left|a_{S}(t)\right|^{2} d t+\left(\Gamma_{D}+\Gamma_{W}\right) \int_{0}^{T}\left|a_{D}(t)\right|^{2} d t} .
$$

In the static steady state case, the efficiency reads [5]

$$
\eta=\frac{\Gamma_{W}\left|a_{D}\right|^{2}}{\Gamma_{S}\left|a_{S}\right|^{2}+\left(\Gamma_{D}+\Gamma_{W}\right)\left|a_{D}\right|^{2}} .
$$

As can be seen from Eq. (11), in order to maximize $\eta$, one should reduce the time that the energy stays in the source coil. This cannot be obtained in the resonant (static) case, because the energy oscillates back and forth between the source and the drain coils. Nevertheless, in the AP mechanism, there is only one transition between the source and the device, so it should be chosen to be as early as possible. The AP dynamics (or any other time-dependent dynamics) should also repeat itself after some repetition time $T_{r e p}$. This is illustrated in Fig. 3. The time scale is of the order of several "loss times" (equal to $\left.1 / \Gamma_{S, D, W}\right)$, in this way we ensure that each cycle of coil charging begins, after the consumption of all the energy from the previous cycle, otherwise interference appears, which will be difficult to be predicted analytically. For the propose AP technique we assume that energy is instantly loaded into the source coil without loss in the beginning of each cycle.

Another important measurement is the amount of energy transferred from the source coil to the device, which is the useful energy consumed as a function of time. This measurement is in fact equal to the nominator in Eq. (11). 


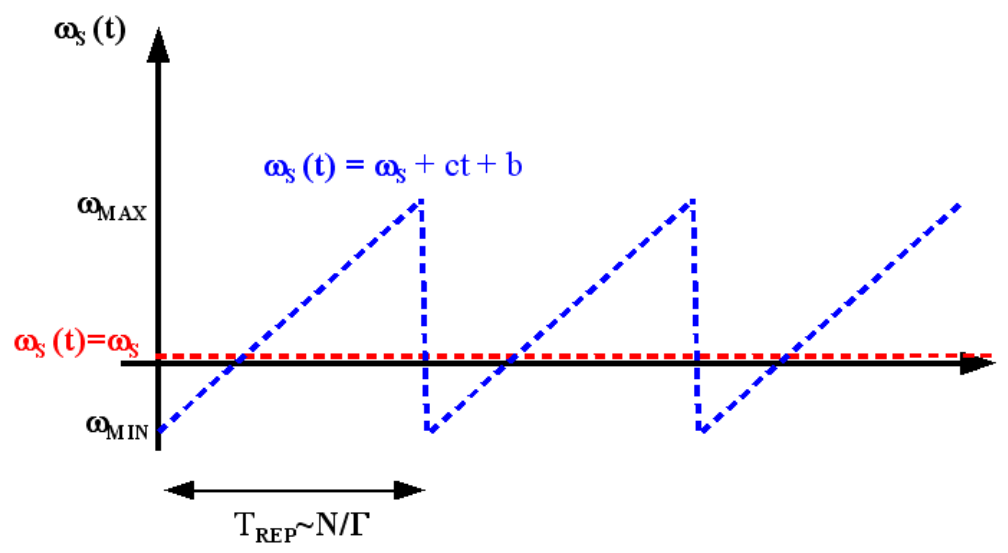

Figure 3: (Color online) The function of the resonant frequency of the source coil. The static case is shown in red, and the repeated linear case (which is the simplest realization or the AP dynamics) is shown in blue.

\section{Simulations}

In order to compare AP and the static mechanisms for energy transfer, several sets of simulations were performed, measuring both conversion efficiency and total energy consumed by the device:

- Comparison between efficiencies as a function of the detuning, for different distances between the coils (determined by the ratio $\kappa / \Gamma_{S, D}$ );

- Influence of variations in the coupling and the loss coefficients;

- Robustness of the adiabatic energy transfer, for time-dependent coupling coefficient.

First, the effect of different resonant frequency between the source coil and the device coil on the total wireless transfer efficiency were examined. For that we changed only the resonance frequency of the device in each numerical run, for two different distances between the coils: 0.8 meter and 1 meter between the coils (where we used the same units and notations as reported in Ref. [5]). Fig. 4 shows that AP is less sensitive to the static detuning $\delta$. As can be shown, the maximal conversion efficiency is achieved for the resonance approach is symmetric about zero detuning, while AP is asymmetric with its maximal efficiency value shifted toward the positive detuning. The explanation is that then the energy transfer occurs at early stage and therefore the energy stays less time in the source coil.

Next, we examine the effect of different coupling and loss coefficients. Fig. 5 shows contour plots of the efficiency coefficient $\eta$ as a function of the coupling $\kappa_{0}$ and the loss rate of the source and the drain coils (where we assumed $\Gamma_{S}=\Gamma_{D}$ ), where we assume fix values of $\Gamma_{W}$ (i.e. cannot be changed for different value 

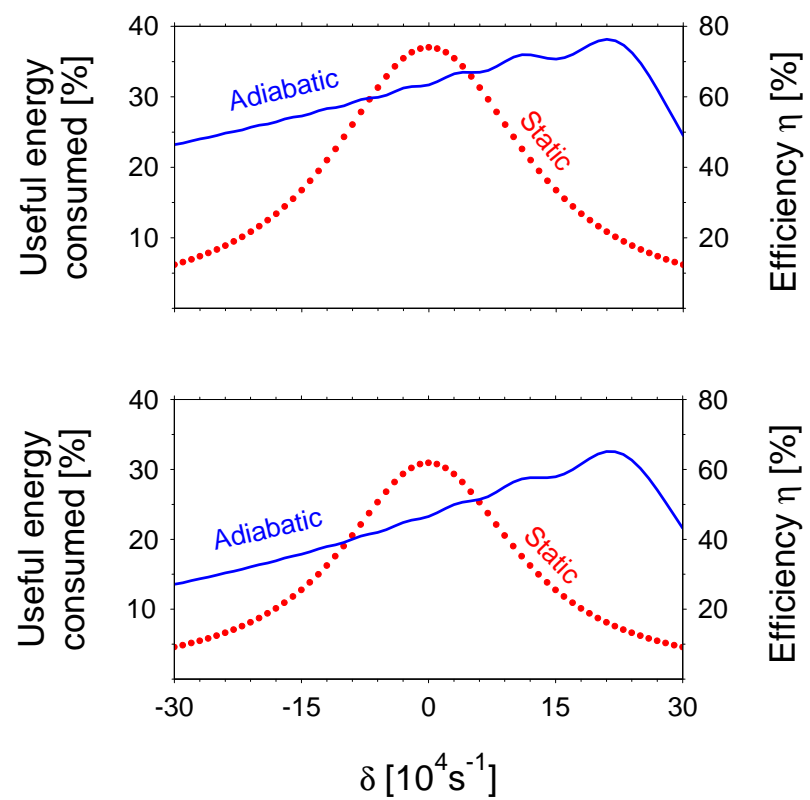

Figure 4: (Color online) Efficiency coefficient $\eta$ (right y-axes) and the useful energy consumed (left y-axes) as a function of the static detuning. The blue solid line depicts AP and the red dotted line is for the static method. The functions $\kappa(t)$ and $\Delta(t)$ are given by Eqs. (9) for the static method and (10) for AP, with the following parameter values: (top frames) $\kappa_{0}=5 \times 10^{4}$ $\mathrm{s}^{-1}, \beta=3 \times 10^{9} \mathrm{~s}^{-2}, \Gamma_{W}=10^{4} \mathrm{~s}^{-1}, \Gamma_{S}=\Gamma_{D}=\kappa_{0} / 30=0.17 \times 10^{4} \mathrm{~s}^{-1}$. (bottom frames) $\kappa_{0}=5 \times 10^{4} \mathrm{~s}^{-1}, \beta=3 \times 10^{9} \mathrm{~s}^{-2}, \Gamma_{W}=10^{4} \mathrm{~s}^{-1}, \Gamma_{S}=\Gamma_{D}=\kappa_{0} / 17=0.29 \times 10^{4} \mathrm{~s}^{-1}$.

of coupling and loss coefficients). The upper frame presents results for the AP technique, while the lower frame is for the static method. AP is obviously more robust to the change in the parameter values compared to the static method. The scheme proposed here, which does not require feedback control, is therefore an alternative to the scheme suggested in 15].

We also wanted to check the effect of time-dependent coupling to the dynamics of energy transfer, which is a more realistic modelling to the process. When the detuning is varied between the two coils, the coupling changes as well (can be inferred from Eq. (3)). The maximal coupling coefficient value is expected to be obtained when the detuning is zero. For that, we chose the following time dependent detuning and coupling coefficient, respectively:

$$
\begin{aligned}
& \Delta(t)=\delta+\beta\left(t-t_{0}\right), \\
& \kappa(t)=\kappa_{0}-\sqrt{|\Delta(t)|},
\end{aligned}
$$

where for our simulations we set $t_{0}=10^{-4} \mathrm{~s}$. Fig. 6 shows the corresponding energy transfer efficiency as a function of time, along with the detuning and coupling. 


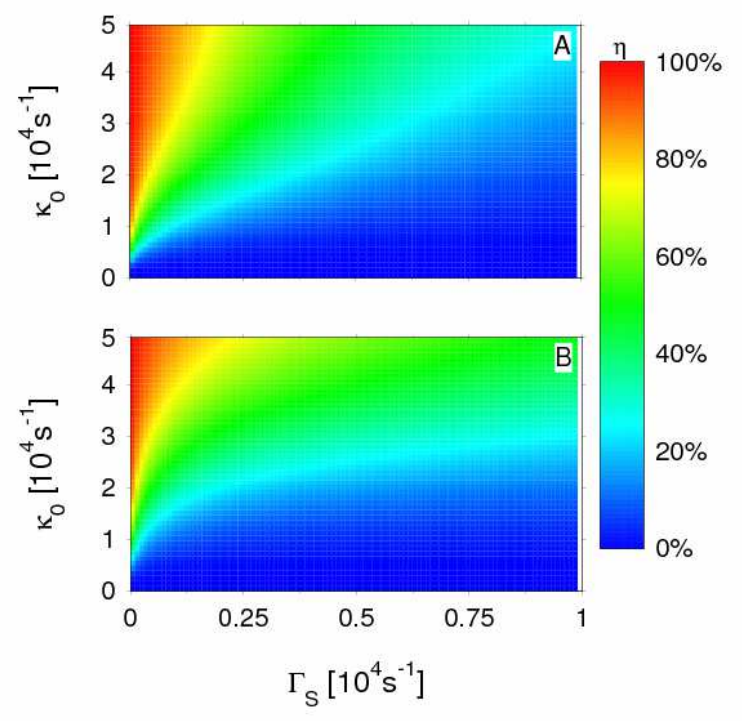

Figure 5: (Color online) Efficiency coefficient $\eta$ for AP (top frame) and the static case (bottom frame) versus the loss rate $\Gamma_{S} \equiv \Gamma_{D}$ and the coupling coefficient $\kappa_{0}$. The functions $\kappa(t)$ and $\Delta(t)$ are given by Eqs. (9) for the static method and (10) for AP, with the following parameter values: $\beta=3 \times 10^{9} \mathrm{~s}^{-2}, \delta=2 \times 10^{5} \mathrm{~s}^{-1}, \Gamma_{W}=10^{4} \mathrm{~s}^{-1}$.

\section{Conclusions}

In conclusion, we have shown that the technique of adiabatic passage, which is well known in quantum optics and nuclear magnetic resonance, has analog in the wireless energy transfer process between two circuites. The factor that enables this analogy is the equivalence of the Schrödinger equation for two-state system, to the coupled-mode equation which describes the interaction between two classical coils in the strong-coupling regime.

The proposed procedure transfers energy wirelessly in effective, robust manner between two coils, without being sensitive to any resonant constraints and noise compared to the resonant scheme demonstrated previously. The application of this mechanism enables efficient energy transfer to several devices as well as optimizing the transfer for several distances and noise interferences.

This work has been supported by the European Commission projects EMALI and FASTQUAST, the Bulgarian NSF grants D002-90/08 and DMU02-19/09 and Sofia University Grant 074/2010. 

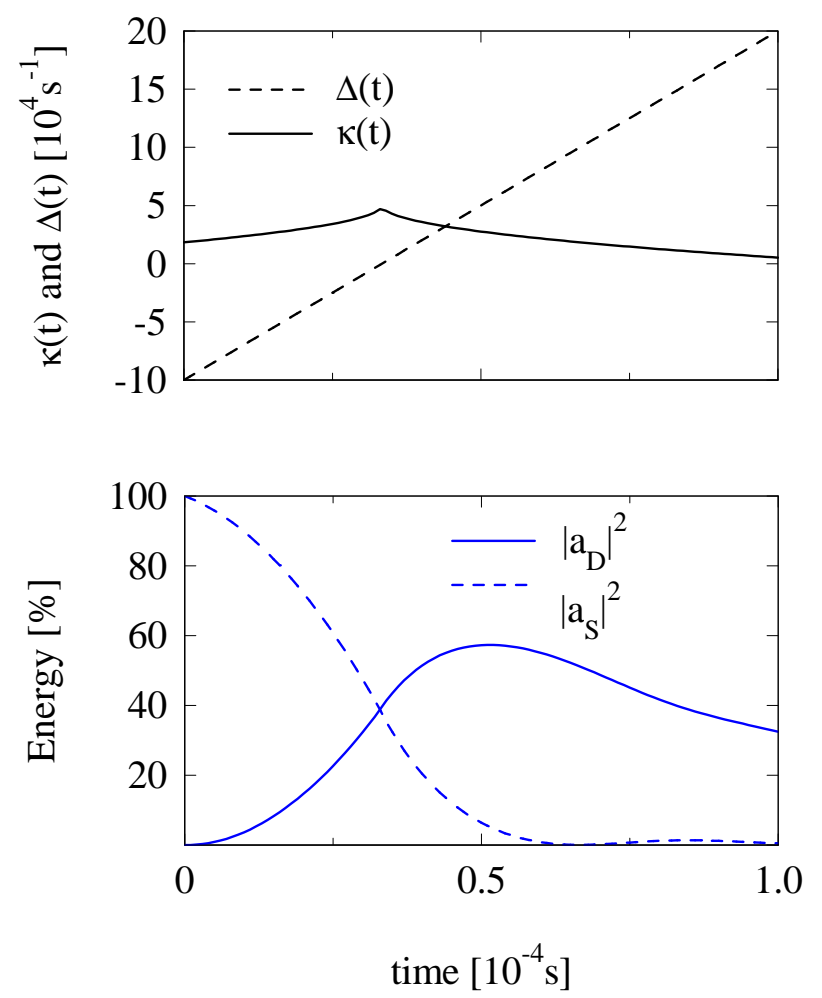

Figure 6: (Color online) Coupling and detuning (top frame) and energy transfer efficiency (lower frame) in AP for the case when the variation of the detuning induces time variation in the coupling; the latter are given by Eqs. (13). We have used the following parameters: $\kappa_{0}=5 \times 10^{4} \mathrm{~s}^{-1}, \beta=3 \times 10^{9} \mathrm{~s}^{-2}, \delta=2 \times 10^{5} \mathrm{~s}^{-1}, \Gamma_{S}=\Gamma_{D}=\Gamma_{W}=3 \times 10^{3} \mathrm{~s}^{-1}$.

\section{References}

[1] Jr. W. Stanley, Induction Coil, US patent number 0349611 issued in September 1886 .

[2] S. Ramo, J. R. Whinnery, T. Van Duzer, Fields and Waves in Communications Electronics (John Wiley and Sons, New York, 1984).

[3] H. Haus, Waves and Fields in Optoelectronics (Prentice-Hall, Englewood Cliffs, NJ, 1984).

[4] W. C. Brown, IEEE Transactions on Microwave Theory and Techniques 32, 1230 (1984).

[5] A. Kurs, A. Karalis, R. Moffatt, J. D. Joannopoulos, P. Fisher, and M. Soljačić, Science 317, 83 (2007).

[6] A. Karalis, J. D. Joannopoulos, and M. Soljačić, Ann. Phys. 323, 34 (2008). 
[7] R. E. Hamam, A. Karalis, J. D. Joannopoulos, and M. Soljačić, Ann. Phys. 324, 1783 (2009).

[8] L. Allen and J. H. Eberly, Optical Resonance and Two-Level Atoms (Dover, New York, 1987).

[9] N. V. Vitanov, T. Halfmann, B. W. Shore, and K. Bergmann, Annu. Rev. Phys. Chem. 52, 763 (2001).

[10] A. Abragam, The Principles of Nuclear Magnetism (Oxford University Press, Oxford, 1961).

[11] C. P. Slichter, Principles of Magnetic Resonance (Springer, Berlin, 1990).

[12] H. Suchowski, D. Oron, A. Arie, Y. Silberberg, Phys. Rev. A 78, 063821 (2008).

[13] H. Suchowski, V. Prabhudesai, D. Oron, A. Arie, Y. Silberberg, Opt. Exp. 17, 12731 (2009).

[14] A. Messiah, Quantum Mechanics (Wiley, New York, 1962).

[15] A. Karalis, Novel photonic phenomena in nanostructured material systems with applications and Mid-Range efficient insensitive Wireless EnergyTransfer (ScD Thesis, Massachusetts Institute of Technology, 2008). 